\title{
Optimization of a Lattice-Based Constellation for Signaling Over a Partial Response Channel
}

\author{
A. K. Khandani and P. Kabal
}

\begin{abstract}
This letter discusses the problem of optimizing a lattice-based signal constellation for signaling over a partial response channel. The objective is to minimize the probability of error which is determined by the combined effects of the additive Gaussian noise and channel memory.
\end{abstract}

Index Terms-Lattice, partial response signaling, TCM.

\section{INTRODUCTION}

A SIGNAL constellation is a finite subset of points from a given lattice packing bounded within a shaping region [1], [2]. The objective in the design of a signal constellation is to minimize the probability of error for given rate, given number of dimensions, and given average energy. This letter discusses the problem of optimizing a lattice-based signal constellation for signaling over a partial response channel.

A channel with a nonflat frequency response (e.g., a partial response channel) results in intersymbol interference (ISI) between successive transmissions. There are two main approaches to handle the problem of ISI, namely: 1) spectral shaping and 2) precoding; channel equalization. This work belongs to the first category.

Coding schemes in which spectral nulls are created in the power spectrum to resemble the channel frequency response are dicussed in [3] and [4]. Run length codes to shape the power spectrum of the transmitted signal are made use of in [5]. The problem of designing codes specifically for the channels with ISI is discussed in [6] and [7]. The problem of designing a constellation for signaling over a set of dimensions with nonequal noise powers is addressed in [8]-[10].

We discuss two methods to directly minimize the probability of error in a lattice-based signal constellation used over a partial response channel. In the first method the minimum distance-to-noise ratio along all of the subspaces is the same (which is the assumption in the conventional systems). In the second method this restriction is relaxed.

\section{StRucture of the Coding AND TRANSMission System}

We use the same transmission system as in [11], where each block is composed of $L$ time intervals and channel has a memory of $S$ symbols. To delete the ISI between blocks,

Paper approved by S. B. Wicker, the Editor for Coding Theory and Techniques of the IEEE Communications Society. Manuscript received August 8, 1995; revised December 12, 1997. This work was supported by Natural Science and Engineering Council (NSERC) of Canada.

A. K. Khandani is with the Department of Electrical and Computer Engineering, University of Waterloo, Waterloo, Ont. N2L 3G1, Canada (email:khandani@shannon.uwaterloo.ca).

P. Kabal is with the Department of Electrical Engineering, McGill University, Montreal, P. Q. H3A 2A7, Canada.

Publisher Item Identifier S 0090-6778(98)05176-9.
TABLE I

Average Energy per Two Dimensions as a Function of the Allocated Rate for a Minimum Distance of One, $M=8$

\begin{tabular}{ccccccc}
\hline$r$ & 4 & 5 & 6 & 7 & 8 & 9 \\
\hline$A(r)$ & 3.0 & 5.9 & 11.9 & 23.6 & 47.8 & 94.6 \\
\hline
\end{tabular}

the last $S$ symbols in each block are set to zero. From the rest $L-S$ dimensions, an appropriate subset is used as a basis for the constellation. We consider the $1 \pm D$ channels [12] and Fourier basis. For the Fourier basis, the noise power of the dimensions will be in pair equal (each such pair is called a two-dimensional (2-D) subchannel). The nonempty 2-D subchannels are divided into $K$ coding schemes, each of dimension $M$. Each coding subsystem uses an independent trellis-coded modulation (TCM) scheme. The noise power, the minimum square distance, the minimum distance-to-noise ratio, and the average energy of the $j$ th subconstellation within the $k$ th coding scheme are shown by $N_{j}^{k}, D_{j}^{k}, P_{j}^{k}=D_{j}^{k} / N_{j}^{k}$, and $E_{j}^{k}$, respectively.

Using the method of [11] to transmit $r_{0}$ bits in an $M=2 m$ dimensional TCM scheme, a signal constellation with $2^{r_{0}+1}$ points is needed. To construct the constellation, the 2-D subconstellations are divided into an inner group and an outer group. The number of points in the inner group of the $j$ th subconstellation is equal to $2^{r_{j}}$, where $\sum_{j} r_{j}=r_{0}$. The number of points in the outer group is $1 / m$ of that in the inner group. The $M$-dimensional ( $M$-D) constellation is constructed by concatenating $m$ such 2-D subconstellations and excluding the $M$-D points corresponding to more than one 2-D outer point [11].

For each 2-D subconstellation, the inner group is used $M-1$ times as often as the outer group. This means that the average energy per two dimensions is equal to $(M-1) / M$ times the average energy of the inner group plus $1 / M$ times the average energy of the outer group. Table I shows the average energy per two dimensions $A$ for $M=8$ and for a minimum distance of one as a function of rate.

The resulting $M$-D constellation is partitioned into the cosets of a baseline lattice. We assume that the dominant error event is the one within a coset. This is the case in most of TCM schemes. The numerical examples are based on the lattice $E_{8}$.

\section{ERror Probability AND EfFective Coding Gain}

For an additive Gaussian noise of power $\sigma^{2}$, the probability of error between two points with distance $d$ is approximately equal to [14. pp. 84, 250]

$$
p_{0} \simeq \frac{1}{2} \exp \left(-d^{2} / 8 \sigma^{2}\right) .
$$


Using (1) in conjunction with the union bound, the probability of error within a lattice $\Lambda$ is computed as

$$
P_{e} \simeq \frac{1}{2} \Theta_{\Lambda}\left[\exp \left(-1 / 8 \sigma^{2}\right)\right]-\frac{1}{2}
$$

where $\Theta_{\Lambda}$ is the weight distribution of $\Lambda$ [14, p. 102].

Using the trellis diagram of the lattice, it can be shown that the weight distribution of the scaled $E_{8}$ lattice is equal to

$$
\begin{aligned}
\Theta_{E_{8}}(q)= & \theta_{3}^{2}\left(q_{0}\right) \theta_{3}^{2}\left(q_{1}\right) \theta_{3}^{2}\left(q_{2}\right) \theta_{3}^{2}\left(q_{3}\right) \\
& +\theta_{2}^{2}\left(q_{0}\right) \theta_{2}^{2}\left(q_{1}\right) \theta_{3}^{2}\left(q_{2}\right) \theta_{3}^{2}\left(q_{3}\right) \\
& +\theta_{2}^{2}\left(q_{0}\right) \theta_{3}^{2}\left(q_{1}\right) \theta_{2}^{2}\left(q_{2}\right) \theta_{3}^{2}\left(q_{3}\right) \\
& +\theta_{2}^{2}\left(q_{0}\right) \theta_{3}^{2}\left(q_{1}\right) \theta_{3}^{2}\left(q_{2}\right) \theta_{2}^{2}\left(q_{3}\right) \\
& +\theta_{3}^{2}\left(q_{0}\right) \theta_{2}^{2}\left(q_{1}\right) \theta_{2}^{2}\left(q_{2}\right) \theta_{3}^{2}\left(q_{3}\right) \\
& +\theta_{3}^{2}\left(q_{0}\right) \theta_{2}^{2}\left(q_{1}\right) \theta_{3}^{2}\left(q_{2}\right) \theta_{2}^{2}\left(q_{3}\right) \\
& +\theta_{3}^{2}\left(q_{0}\right) \theta_{3}^{2}\left(q_{1}\right) \theta_{2}^{2}\left(q_{2}\right) \theta_{2}^{2}\left(q_{3}\right) \\
& +\theta_{2}^{2}\left(q_{0}\right) \theta_{2}^{2}\left(q_{1}\right) \theta_{2}^{2}\left(q_{2}\right) \theta_{2}^{2}\left(q_{3}\right) \\
& +8 \theta_{2}\left(q_{0}\right) \theta_{2}\left(q_{1}\right) \theta_{2}\left(q_{2}\right) \theta_{2}\left(q_{3}\right) \\
& \times \theta_{3}\left(q_{0}\right) \theta_{3}\left(q_{1}\right) \theta_{3}\left(q_{2}\right) \theta_{3}\left(q_{3}\right), \\
& \quad q_{j}=q^{4 s_{j}^{2}}, \quad j=0,1,2,3
\end{aligned}
$$

where $\theta_{2}, \theta_{3}$ are the Jacobi theta functions (refer to [13] for definition) and $s_{j}$ denotes the scale factor along the $j$ th 2 -D subspace. We truncate the weight distribution to the set of the 240 nearest neighbors of the $E_{8}$ lattice. This results in

$$
\begin{aligned}
P_{e} \simeq & F\left(Z_{j}^{k}, j=0,1,2,3\right) \\
\equiv & \frac{1}{2}\left(4 Z_{0}^{4}+4 Z_{1}^{4}+4 Z_{2}^{4}+4 Z_{3}^{4}+16 Z_{0}^{2} Z_{1}^{2}+16 Z_{0}^{2} Z_{2}^{2}\right. \\
& \quad+16 Z_{0}^{2} Z_{3}^{2}+16 Z_{1}^{2} Z_{2}^{2}+16 Z_{1}^{2} Z_{3}^{2}+16 Z_{2}^{2} Z_{3}^{2} \\
& \left.\quad+128 Z_{0} Z_{1} Z_{2} Z_{3}\right)
\end{aligned}
$$

where $Z_{j}=\exp \left(-d_{j}^{2} / 2 \sigma_{j}^{2}\right)$. The effective gain of the system $\gamma_{e}$ is defined as the saving in energy with respect to a reference system with the same rate and probability of error. The reference system has a rate of $R_{0} / L$ bits per dimension, where $R_{0}$ is the total rate in bits per signaling interval. For the reference system, we assume continuous approximation and use (1) for the error probability.

\section{Minimizing the Probability of ERRor}

Conventionally, the system is designed to have equal protection for all the dimensions, i.e., $P_{j}^{k}=\bar{P}, \forall j, k$. In this case it is easy to show that

$$
\bar{P}=\frac{E_{0}}{\sum_{k=0}^{K-1} \sum_{j=0}^{m-1} A\left(R_{j}^{k}\right) N_{j}^{k}}
$$

where $E_{0}$ is the overall average energy and $A(\cdot)$ is given in Table I. This results in the following optimization problem:

$$
\begin{cases}\text { Minimize } & \sum_{k=0}^{K-1} \sum_{j=0}^{m-1} A\left(R_{j}^{k}\right) N_{j}^{k} \\ \text { Subject to } & \sum_{k=0}^{K-1} \sum_{j=0}^{m-1} R_{j}^{k}=R_{0}, \quad R_{j}^{k} \geq \log _{2}(m) .\end{cases}
$$

This is solved by the following algorithm.

1) Set $R_{j}^{k}=\log _{2}(m), \forall j, k$. It remains $\left[R_{0}-K m \log _{2}(m)\right]$ bits to be distributed.

2) Allocate 1 bit to the 2-D subconstellation which has the smallest value for the quantity $\left[A\left(R_{j}^{k}+1\right)-A\left(R_{j}^{k}\right)\right] N_{j}^{k}$. Update the rates. If there are still bits to be distributed go to step 2, otherwise quit.

As an alternative to (6), we can directly minimize the error probability, i.e.,

$$
\left\{\begin{aligned}
\text { Minimize } & \sum_{k=0}^{K-1} F\left(Z_{j}^{k}, j=0,1,2,3\right), \\
\text { Subject to } & \sum_{j=0}^{k}=\exp \left(-D_{j}^{k} / 2 N_{j}^{k}\right) \\
& \sum_{k=0}^{K-1} \sum_{j=0}^{k-1}=R_{0}, \quad R_{j}^{k} \geq \log _{2}(m) \\
& E_{j}^{k-1}=E_{0}, \quad E_{j}^{k} \geq 0 .
\end{aligned}\right.
$$

This optimization problem is solved using the following twostep iterative procedure.

\section{A. Finding the Rate Distribution for a Given Energy Distribution}

1) Set $R_{j}^{k}=\log _{2}(m), \forall j, k$. It remains $\left[R_{0}-K m \log _{2}(m)\right]$ bits to be distributed.

2) Allocate one bit to the subchannel which by receiving it will result in the least increase in the objective function. Update the rates. If there are still bits to be distributed go to step 2, otherwise quit.

\section{B. Finding the Energy Distribution for a Given Rate Distribution}

The objective function in (7) is convex $\cup$. As a result, the optimum point over the convex region determined by the energy constraint is determined by the Lagrange method. This results in

$$
\left\{\begin{array}{l}
Z_{j}^{k} \frac{\partial}{\partial Z_{j}^{k}}\left[F\left(Z_{j}^{k}, j=0,1,2,3\right)\right]=\lambda N_{j}^{k} A\left(R_{j}^{k}\right) \\
\sum_{k=0}^{K-1} \sum_{j=0}^{m-1} E_{j}^{k}=E_{0} .
\end{array}\right.
$$

This set is solved by an iterative method.

\section{Assignment of Subconstellations to Subchannels}

1) Arrange the nonempty subchannels according to the value of the noise power and the 2-D subconstellations according to the value of $P_{j}^{k}$.

2) Assign the members of these two sets with the same order to each other.

\section{NUMERICAL RESULTS}

We consider a coding scheme with parameters $L=29,31$, $K=3, M=8$ (using lattice $E_{8}$ ), and $R_{0}=36$ bits per signaling interval. Over a flat channel, the corresponding 


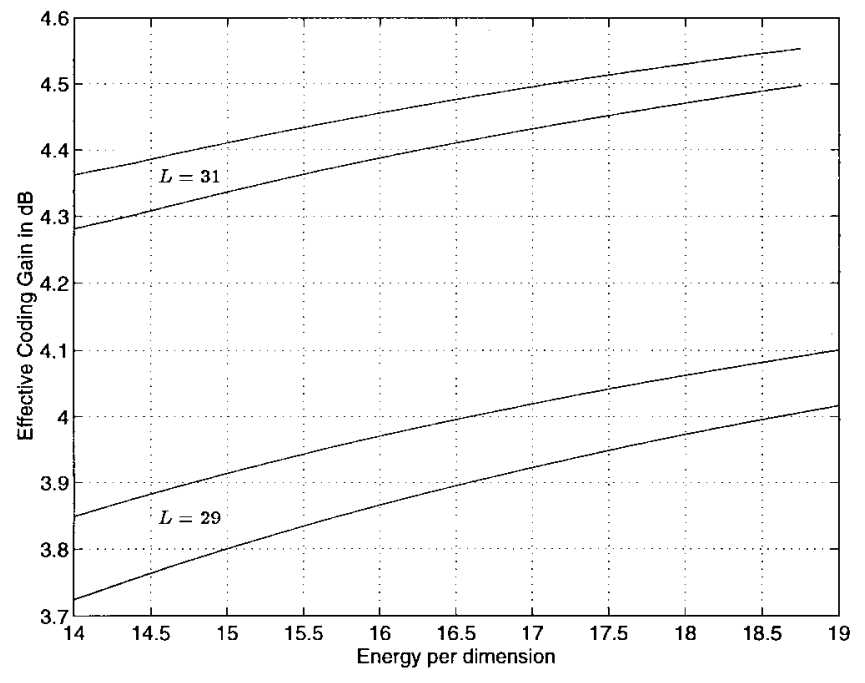

Fig. 1. Effective gain as a function of the energy per dimension for the two proposed methods, for $L=29,31, K=3$, and $R_{0}=36$ bits per signaling interval (probability of error is in the rage of $10^{-3}-10^{-6}$ ).

TCM scheme results in an asymptotic coding gain of 5.41 $\mathrm{dB}$ [11]. Unlike our analysis, this gain is based on the minimum distance and does not include the effect of the error multiplicity. We apply both of our design methods to this problem. The performance is measured in terms of the effective gain $\gamma_{e}$ for a probability of error in the range of $10^{-3}-10^{-6}$ (as a function of the energy per dimension). This is shown in Fig. 1. The second method outperforms the first one in terms of $\gamma_{e}$ (or, equivalently, in terms of error performance) without any increases in the complexity.

\section{REFERENCES}

[1] G. D. Forney Jr. and L. F. Wei, "Multidimensional constellations-Part I: Introduction, figures of merit, and generalized cross constellations," IEEE J. Select. Areas Commun., vol. 7, pp. 877-892, Aug. 1989.

[2] A. K. Khandani and P. Kabal, "Shaping multi-dimensional signal spaces-Parts I, II: Optimum shaping, shell mapping," IEEE Trans. Inform. Theory, vol. 39, pp. 1799-1819, Nov. 1993.

[3] G. D. Forney and A. R. Calderbank, "Coset codes for partial response channels, or, coset codes with spectral nulls," IEEE Trans. Inform. Theory, vol. 35, pp. 925-943, Sept. 1989.

[4] A. R. Calderbank and J. E. Mazo, "Baseband line codes via spectral factorization,” IEEE J. Select. Areas Commun., vol. 7, pp. 914-928, Aug. 1989.

[5] R. Karabed and P. H. Siegel, "Matched spectral null trellis codes for partial response channels," Int. Symp. Information Theory, Kobe, Japan, June 1988, pp. 142-143.

[6] J. K. Wolf and G. Ungerboeck, "Trellis coding for partial response channels," IEEE Trans. Commun., vol. COM-34, pp. 765-773, Aug. 1986.

[7] J. Lechleider, "The optimum combination of block codes and receivers for arbitrary channels," IEEE Trans. Commun., vol. 38, pp. 615-621, May 1990.

[8] S. Kasturia, J. T. Aslanis, and J. M. Cioffi, "Vector coding for partial response channels," IEEE Trans. Inform. Theory, vol. 36, pp. 741-762, July 1989.

[9] M. L. Honig, K. Steiglitz, and S. Norman, "Optimization of signal sets for partial-response channels-Part I: Numerical techniques," IEEE Trans. Inform. Theory, vol. 37, pp. 1327-1341, Sept. 1991.

[10] M. L. Honig, "Optimization of signal sets for partial-response channels-Part II: Asymptotic coding gain," IEEE Trans. Inform. Theory, vol. 37, pp. 1342-1354, Sept. 1991.

[11] L. F. Wei, "Trellis coded modulation with multidimensional constellations," IEEE Trans. Inform. Theory, vol. IT-33, pp. 483-501, July 1987.

[12] P. Kabal and S. Pasupathy, "Partial-response signaling," IEEE Trans. Commun., vol. COM-23, pp. 921-934, Sept. 1975.

[13] J. H. Conway and N. J. A. Sloane, Sphere Packings, Lattices and Groups. New York: Springer-Verlag, 1988

[14] J. M. Wozencraft and I. M. Jacobs, Principles of Communication Engineering. New York: Wiley, 1965. 